\title{
EXACTLY RENORMALIZABLE MODEL IN THE QUANTUM THEORY OF FIELDS
}

\author{
by TH. W. RUIJGROK and L. VAN HOVE
}

Instituut voor theoretische Fysica der Rijksuniversiteit, Utrecht, Nederland

\begin{abstract}
Synopsis
A simple model is considered for the interaction of a boson field with infinitely heavy nucleons, susceptible of an exact determination of the physical nucleon states and of the renormalization constants. It incorporates as special cases the Lee model and the neutral scalar field in scalar interaction with heavy nucleons. The renormalized coupling constants remain different from zero for infinite cut-off although part of the physical effects of the coupling vanish in this limit. The system becomes then essentially equivalent to the neutral scalar field in scalar interaction with heavy nucleons, which gives vanishing boson-nucleon scattering but non-vanishing nucleon-nucleon interaction.
\end{abstract}

1. Introduction. T. D. Lee has proposed recently ${ }^{1)}$ a very interesting model of renormalizable field theory, simple enough to permit an exact calculation of mass and charge renormalization as well as of some physical processes, but still sufficiently rich in non trivial properties to be quite suggestive for the general discussion of the basic difficulties of quantum electrodynamics and meson theory. Lee's model consists of a nucleon capable of two internal states (the $N$ - and $V$-particles), and of a field of relativistic neutral spinless bosons (the $\theta$-particles), with an interaction allowing exclusively for the transitions

$$
N+\theta \rightleftarrows V
$$

Whereas for this model the physical vacuum and the physical $N$-particle are identical with the corresponding bare (i.e. no-interaction) states, the physical $V$-particle differs from the bare $V$-particle. Its wave fuction however is very simple and can be written in closed form, providing the exact value for the $V$-particle mass renormalization and the renormalization constant $Z_{2}$ (we use the notation of Dyson ${ }^{2}$ ) for all renormalization constants). Since the model does not give rise to vertex diagrams nor to nucleon pair creation, the renormalization constants $Z_{1}$ and $Z_{3}$ are trivially equal to unity. Consequently knowledge of $Z_{2}$ permits exact renormalization of the coupling constant. As shown by Lee, still another expression can be 
obtained in closed form, namely the general stationary state of $\theta-N$ scattering.

The Lee model exhibits the remarkable property that the renormalized coupling constant $g$ approaches zero for an infinite cut-off (i.e. in the limit of a point nucleon), whatever the value is of the unrenormalized coupling constant $g^{0}$. It was further shown by Källén and $\mathrm{Pauli}{ }^{3}$ ) that if one required nonetheless a non-vanishing $g$ for a point nucleon one is forced to admit negative probabilities into the theory. The vanishing of $g$ for infinite cut-off in this simple model is all the more interesting that the possibility of a similar behaviour has been suggested on other grounds by Landau, Abrikosov and Khalatnikov for the renormalized charge in quantum electrodynamics ${ }^{4}$ ).

A weakness of the Lee model is the impossibility of successive emission or absorption of several bosons. Apart from preventing the occurrence of vertex diagrams and giving consequently the trivial value unity to the renormalization constant $Z_{1}$, this circumstance implies that the model has essentially a finite number of degrees of freedom. Still one of the most characteristic features of realistic fields is of course their infinite number of degrees of freedom and one would like to have it incorporated in the simple models used for the study of basic field-theoretical difficulties. A model incorporating this feature has long been known: it is composed of a scalar neutral Bose field coupled to infinitely heavy nucleons through the normal scalar interaction 5). This interaction allows for successive emission and absorption of unlimited numbers of bosons, thus bringing into play the infinity of degrees of freedom of the boson field. It does not give rise to meson-nucleon scattering, but manifests itself physically through a Yukawa potential between nucleons. Although instructive for other purposes ${ }^{6}$ ) the model just mentioned has the great disadvantage of giving no coupling constant renormalization. It is therefore of very limited interest for renormalization studies.

The model to be presented briefly in the present note ${ }^{*}$ ) combines the occurrence of coupling constant renormalization with the possibility of successive emission and absorption of an unrestricted number of field particles. The constants $Z_{1}$ and $Z_{2}$ are both different from unity while one has still $Z_{3}=1$ because no pair processes are included. Although formally much more complicated than the Lee model and the scalar neutral field mentioned above**), our model retains the property that the physical particle states can be obtained in closed form, thus providing the exact value

*) A detailed study will be published later by one of us (Th. W. R.).

**) The mathematical structure of Lee's model is actually, at least for the $N-\theta$-system, that of a well known case of exactly soluble eigenvalue problem. It was already used by Dirac ?) with a slightly different choice of parameters as model for resonance reactions. As for the scalar neutral field in scalar interaction with static nucleons, it is well known to reduce mathematically to a very elementary harmonic oscillator problem. 
of all renormalization constants. No closed solution seems to exist for physically observable processes (except for infinite cut-off). The model consists of a field of relativistic neutral spinless bosons (following Lee we call them $\theta$-particles) interacting with infinitely heavy nucleons, each of which is capable of $n>1$ internal states called $V_{q}$ where the index $q$ runs over the integers modulo $n$ (thus $V_{q}$ is identical with $V_{q+n}$ for all $q$ ). The interaction gives rise to the following transitions

$$
V_{q} \rightleftarrows V_{q+1}+\theta
$$

(the sum $q+1$ is to be understood in the algebra of integers modulo $n$ ) with a strength depending on $q$ (i.e. on the internal nucleon state) and measured by a coupling constant $g_{q}^{0}$ (the index $q$ of $g_{q}^{0}$ also runs over the integers modulo $n$ ). This model reduces to the Lee model when all $g_{q}^{0}$ but one vanish, to the scalar neutral field in scalar interaction with static nucleons when all $g_{q}^{0}$ are equal.

As will be seen hereafter, the most striking property of the new model is the following. The renormalized coupling constants $g_{q}\left(g_{q} \neq g_{q}^{0}\right)$, while being different for finite cut-off, reduce in the limit of point nucleons to one and the same value $g=\left(g_{1}^{0} \ldots g_{n}^{0}\right)^{1 / n}$. This suggests that in this limit our model essentially degenerates into the scalar interaction case: thus the $\theta-V_{q}$ scattering cross section, different from zero for a finite cut-off, is expected to vanish in the limit of an infinite cut-off, but a $V_{q}-V_{q}$, interaction will remain even in this limit. These properties have been established to second non vanishing order in the coupling constants for $\theta-V_{\boldsymbol{q}}$ and $V_{q}-V_{q^{\prime}}$ scattering, but undoubtedly hold to arbitrary order. It is interesting to compare them with the behaviour of the Lee model and the suggestion of Landau for quantum electrodynamics: whereas in the latter cases all effects of the interaction vanish (or are suggested to vanish) for infnite cut-off, in the model here presented part of the interaction effects vanish but part of them remain, and the normalized coupling is always measurable.

The hamiltonian of the new model is given in the following section, where the physical state of the $V_{q}$-particle and the renormalization constants are also determined. Section 3 is devoted to a brief consideration of the $V_{q}-\theta$ and the $V_{q}-\mathrm{V}_{q}$, interaction.

2. The hamiltonian and the physical nucleon states. The hamiltonian of our model has the following form:

$$
\left.\begin{array}{c}
H=\sum_{q=1}^{n} \sum_{\boldsymbol{p}}\left(m-\delta m_{q}\right) \psi_{q}^{*}(\boldsymbol{p}) \psi_{q}(\boldsymbol{p})+\sum_{\boldsymbol{k}} \omega(k) a^{*}(\boldsymbol{k}) a(\boldsymbol{k}) \\
-(2 v)^{-1 / 2} \sum_{\boldsymbol{p}^{\prime}=\boldsymbol{p}+\boldsymbol{k}} \sum_{q=1}^{n} g_{\boldsymbol{q}}^{0} \omega^{-1 / 2}(k) f(k)\left[\psi_{q+1}^{*}(\boldsymbol{p}) \psi_{q}\left(\boldsymbol{p}^{\prime}\right) a^{*}(\boldsymbol{k})+\text { c.c. }\right]
\end{array}\right\}
$$

The creation and annihilation operators for a nucleon of internal state $V_{q}$ and momentum $\boldsymbol{p}$ are $\psi_{q}^{*}(\boldsymbol{p})$ and $\psi_{q}(\boldsymbol{p})$. The corresponding operators for 
the $\theta$-particles are $a^{*}(\boldsymbol{k}), a(\boldsymbol{k})$. The usual commutation and anti-commutation relations hold, i.e.

$$
\left[a(\boldsymbol{k}), a^{*}(\boldsymbol{k})\right]=\left\{\psi_{q}^{*}(\boldsymbol{p}), \psi_{q}(\boldsymbol{p})\right\}=1,\left[a(\boldsymbol{k}), \psi_{q}(\boldsymbol{p})\right]=\left[a^{*}(\boldsymbol{k}), \psi_{q}(\boldsymbol{p})\right]=0,
$$

while the other commutators of $a(\boldsymbol{k}), a^{*}\left(\boldsymbol{k}^{\prime}\right)$ and the other anticommutators of $\psi_{q}(\boldsymbol{p}), \psi_{q}^{*}\left(\boldsymbol{p}^{\prime}\right)$ vanish. $\omega(k)=\left(k^{2}+\mu^{2}\right)^{1 / 2}$ is the energy of a $\theta$ particle of momentum $\boldsymbol{k}$ (we put $\hbar=c=1$ ). The observable (i.e. renormalized) energy of a nucleon is assumed to be independent of the internal state $q$ and of the momentum. It is denoted by $m$. The quantity $\delta m_{q}$,. independent of momentum, is the mass renormalization term for a nucleon in state $q$. The function. $f(k)$ is a cut-off function describing the nucleon shape. One has $f(k)=1$ for all $k$ in the case of point nucleons. $v$ denotes the usual volume of periodicity.

As seen from (1) the number of nucleons is a constant of the motion: no pair creation is allowed for. The bare particle state of total momentum zero with one nucleon in the internal state $q$ and $l_{\boldsymbol{k}_{1}}$ bosons with momentum $\boldsymbol{k}_{1}, \boldsymbol{l}_{\boldsymbol{k}_{2}}$ bosons with momentum $\boldsymbol{k}_{2}$, etc. can conveniently be written in the form $\mid q,\left\{l_{k}\right\}_{n}>$ where $n=\sum_{k} l_{k}$ is the total number of $\theta$-particles present. All but a finite number of the integers $l_{k}$ are zero. The exact wave function for the physical nucleon state $V_{q}$ is the following, as can be verified by insertion in the Schrödinger equation,

$$
\mid \phi_{q}>=N(q)\left[\left|q,\{0\}_{0}>+\sum_{n=1}^{\infty} \sum \alpha_{q}^{(n)}\left(\left\{l_{k}\right\}_{n}\right)\right| q+n,\left\{l_{k}\right\}_{n}>\right]
$$
with

$$
\alpha_{q}^{(n)}\left(\left\{l_{k}\right\}_{n}\right)=(2 v)^{-1 / 2} \Pi_{j=q}^{n+q-1} g_{j}^{0} \Pi_{k}\left(l_{k} !\right)^{-1 / 2}\left[\omega^{-3 / 2}(k) \cdot f(k)\right]^{l_{k}}
$$

In (2) the second summation runs over all sets of $l_{k}$ such that $\Sigma l_{k}=n$. From the requirement that the eigenvalue be $m$ one finds

$$
\begin{aligned}
\delta m_{q}=-(2 y)^{-1 / 2} g_{q}^{0} \sum_{k^{\prime}} \omega^{-1 / 2}\left(k^{\prime}\right) f\left(k^{\prime}\right) \alpha_{q}^{(1)}\left(\left\{\delta_{k k^{\prime}}\right\} 1\right)= \\
=-(2 v)^{-1}\left(g_{q}^{0}\right)^{2} \Sigma_{k} \omega^{-2}(k) \cdot f^{2}(k) .
\end{aligned}
$$

It may be noted that, contrary to what is the case in the Lee-model, all nucleon states are now affected by the interaction and need mass-renormalization.

The renormalization constant $Z_{2}$ for the nucleon state $V_{q}$, to be denoted by $Z_{2}(q)$, can be obtained from the normalization constant in (2) by the relation

$$
Z_{2}(q)=N^{2}(q) .
$$

That this determination of $Z_{2}(q)$ agrees with the usual definition in terms of bare-particle propagation functions can be checked explicitly in the present case, as was also done in other simple cases ${ }^{8}$ ). From (5) we find

$$
Z_{2}(q)=\left[1+\left(g_{q}^{0}\right)^{2} L+\left(g_{q}^{0} g_{q+1}^{0}\right)^{2}(2 !)^{-1} L^{2}+\left(g_{q}^{0} g_{q+1}^{0} g_{q+2}^{0}\right)^{2}(3 !)^{-1} L^{3}+\ldots\right]^{-1}
$$


where $L=(2 v)^{-1} \Sigma_{k} \omega^{-3}(k) \cdot f^{2}(k)$ is a logarithmically divergent quantity for point nucleons. Bearing in mind that $g_{q+n}^{0}$ is identical to $g_{q}^{0}$ one can sum the series (6) exactly. One finds

$$
\left.\begin{array}{r}
Z_{2}^{-1}(q)=\Theta_{0}(a L)+a^{-1}\left(g_{q}^{0}\right)^{2} \Theta_{1}(a L)+a^{-2}\left(g_{q}^{0} g_{q+1}^{0}\right)^{2} \Theta_{2}(a L)+ \\
\ldots+a^{1-n}\left(g_{q}^{0} g_{q+1}^{0} \ldots g_{q+n-2}^{0}\right)^{2} \Theta_{n-1}(a L)
\end{array}\right\}
$$

where

$$
a=\left(g_{1}^{0} g_{2}^{0} \ldots g_{n}^{0}\right)^{2 / n}
$$

and, putting $\alpha=\exp (2 \pi i / n)$,

$$
\left.\begin{array}{l}
\Theta_{k}(x)=\sum_{j=0}^{\infty} x^{k+j n} /(k+j n) ! \\
=n^{-1}\left\{\exp x+\alpha^{-k} \exp (\alpha x)+\ldots+\alpha^{(1-n) k} \exp \left(\alpha^{n-1} x\right)\right\}
\end{array}\right\}
$$

In particular, for $n=2$ (two internal nucleon states), one simply gets

$$
\Theta_{0}(x)=\cosh x, \Theta_{1}(x)=\sinh x,
$$

and consequently

$$
Z_{2}^{-1}(q)=\cosh \left(g_{1}^{0} g_{2}^{0} L\right)+\left(g_{q}^{0} / g_{q+1}^{0}\right) \sinh \left(g_{1}^{0} g_{2}^{0} L\right) .
$$

Noticing that (8) reduces to $\Theta_{k}(x) \simeq n^{-1} \exp x$ for large $x$, we immediately obtain from (7) the value of $Z_{2}(q)$ for large cut-off

$$
Z_{2}^{-1}(q) \simeq n^{-1} \exp (a L) \cdot\left\{1+a^{-1}\left(g_{q}^{0}\right)^{2}+\ldots+a^{1-n}\left(g_{q}^{0} \ldots g_{q+n-2}^{0}\right)^{2}\right\}
$$

The renormalized coupling constant $g_{q}$ can most easily be derived from the physical particle states by the formula

$$
g_{q}=g_{q}^{0}<\phi_{q+1}\left|\Sigma_{q^{\prime}=1}^{n} \Sigma_{p} \psi_{q^{\prime}+1}^{*}(\boldsymbol{p}) \psi_{q^{\prime}}(\boldsymbol{p})\right| \phi_{q}>
$$

also used in other simple models ${ }^{8}$ ). It gives with (2), (3) and (6)

$$
\begin{gathered}
g_{q}=Z_{2}^{1 / 2}(q) Z_{2}^{1 / 2}(q+1) g_{q}^{0}\left[1+\left(g_{q+1}^{0}\right)^{2} L+\left(g_{q+1}^{0} g_{q+2}^{0}\right)^{2}(2 !)^{-1} L^{2}+\ldots\right] \\
=Z_{2}^{1 / 2}(q) \cdot Z_{2}^{-1 / 2}(q+1) g_{q}^{0}
\end{gathered}
$$

The method of Dyson, based on the consideration of Feynman diagrams, would give

$$
g_{q}=Z_{2}^{1 / 2}(q) \cdot Z_{2}^{1 / 2}(q+1) Z_{1}^{-1}(q) g_{q}^{0}
$$

where $Z_{1}(q)$ is the renormalization constant belonging to a vertex in which a $V_{q}$-particle emits a $\theta$-particle. Comparing (10) and (11) we get

$$
Z_{1}(q)=Z_{2}(q+1)
$$

a relation analogous to the Ward identity of electrodynamics. Explicit calculation of $Z_{1}(q)$ by the Dyson method leads to the same result.

The renormalized coupling constant $g_{q}$ is easily determined in the limit 
of infinite cut-off. Application of (9) gives in this limit

$$
g_{q} \simeq a^{1 / 2}=\left(g_{1}^{0} \ldots g_{n}^{0}\right)^{1 / n}
$$

as announced in the introduction.

3. $V_{q}-\theta$ and $V_{q}-V_{q}$ scattering. The $S$-matrix element for scattering of a $\theta$-particle by a $V_{q}$-particle, with initial and final momenta $\boldsymbol{k}$ and $\boldsymbol{k}^{\prime}$ for the $\theta$-particle, is found to be

$$
\left.\begin{array}{c}
M=\delta_{k k^{\prime}}-i \pi v^{-1} \delta\left[\omega(k)-\omega\left(k^{\prime}\right)\right] \omega^{-2}(k) \cdot f^{2}(k) . \\
\left(g_{q-1}^{2}-g_{q}^{2}\right) \cdot\left[1-(4 \pi)^{-2}\left(g_{q-1}^{2}-g_{q}^{2}\right) \omega(k) \int \omega^{-3}\left(k^{\prime \prime}\right) f^{2}\left(k^{\prime \prime}\right)\left\{\omega\left(k^{\prime \prime}\right)-\omega(k)-i \varepsilon\right\}^{-1} \mathrm{~d} \boldsymbol{k}^{\prime \prime}\right]
\end{array}\right\}
$$

to fourth order in the renormalized coupling constants; $\varepsilon$ is an infinitesimal positive number. This expression holds for arbitrary value of the total momentum. From (13) we get the differential cross section to sixth order

$$
\begin{gathered}
\mathrm{d} \sigma / \mathrm{d} \Omega=(4 \pi)^{-2}\left(g_{q-1}^{2}-g_{q}^{2}\right)^{2} \cdot \omega^{-2}(k) \cdot f^{4}(k) . \\
{\left[1-(2 \pi)^{-3}\left(g_{q-1}^{2}-g_{q}^{2}\right) \omega(k) \int \omega^{-3}\left(k^{\prime \prime}\right) f^{2}\left(k^{\prime \prime}\right)\left\{\omega\left(k^{\prime \prime}\right)-\omega(k)\right\}_{P}^{-1} \mathrm{~d} \boldsymbol{k}^{\prime \prime}\right]}
\end{gathered}
$$

The subscript $P$ denotes that the principal value of the integral has to be taken. As expected (14) vanishes in the limit of a point nucleon, because $g_{q-1}$ and $g_{q}$ are then equal. On the other hand for $g_{q}=0$ this expression agrees to sixth order with the $N-\theta$ scattering cross section for Lee's model.

The interaction between two heavy particles will now be briefly considered. We restrict ourselves to the second order $S$-matrix elements for $V_{q}-V_{q^{\prime}}$ scattering. They are found to be

$$
\left.\begin{array}{l}
M_{1}=(2 \pi)^{-2} i g_{q} g_{q^{\prime}-1} \delta_{4}\left(p_{q}+p_{q^{\prime}}-p_{q+1}-p_{q^{\prime}-1}\right) \omega^{-2}(k) f^{2}(k) \\
M_{2}=(2 \pi)^{-2} i g_{q^{\prime}} g_{q-1} \delta_{4}\left(p_{q}+p_{q^{\prime}}-p_{q-1}-p_{q^{\prime}+1}\right) \omega^{-2}\left(k^{\prime}\right) f^{2}\left(k^{\prime}\right)
\end{array}\right\}
$$

for scattering into the internal states $V_{q+1}-V_{q^{\prime}-1}$ and $V_{q-1}-V_{q^{\prime}+1}$ respectively. The $\delta_{4}$-functions express momentum-energy conservation; $\boldsymbol{k}=\boldsymbol{p}_{q}-\boldsymbol{p}_{q+1}$ and $\boldsymbol{k}^{\prime}=\boldsymbol{p}_{q^{\prime}}-\boldsymbol{p}_{q^{\prime}+1}$ are the momenta of the virtual $\theta$ particles. The matrix elements clearly do not vanish in the limit of infinite cut-off. Their $\boldsymbol{k}$-dependence, being in $\omega^{-2}(k)$, is characteristic of a Yukawa potential between nucleons. The first equation (15) can be applied to the Lee model by putting $q^{\prime}=q+1$; for this case $g_{q}$ and consequently $M_{1}$ reduce to zero for infinite cut-off 9 ).

The authors' thanks are due to Prof. W. Pauli for an illuminating discussion on the Lee model as well as for useful correspondence on early tentative versions of the present model.

Received 25-7-56. 


\section{REFERENCES}

1) Lee, T. D., Phys. Rev. 95 (1954) 1329.

2) Dyson, F. J., Phys. Rev. 75 (1949) 486 and 1736.

3) Källén, G. and Pauli, W., Dan. mat. fys. Medd. 30 (1955) no. 7.

4) Landau, L. D., Abrikosov, A. A. and Khalatnikov, I. M., Dokl. Akad. Nauk S.S.S.R. 95 (1954) 497, 773, 1177, and ibid. 96 (1954) 261.

For a survey of this work, see Landau, L. D., On the quantum theory of fields, in "Niels Bohr and the development of physics", edited by W. Pa uli, Pergamon Press, London (1955), p. 52-69. A similar suggestion concerning electrodynamics has been made by $\mathrm{Pauli}$ (private communication) and was communicated at the Pisa Conference on elementary Particles (June 1955). Still one should keep in mind that the charge renormalization in electrodynamics is of a different nature than in the Lee model (and in the model presented here), because it is essentially determined by pair processes (constant $Z_{3}$ of D y $50 \mathrm{n}$ ).

5) This model is briefly discussed in appendix I of Lee's paper, ref. 1.

6) This model is very suitable for exhibiting the impossibility in the case of point nucleons to expand the physical particle states in bare particle states. See Van Hove, L., Physica 18 (1952) 145, or Wightman, A. S. and Schweber, S. S., Phys. Rev. 98 (1955) 812, esp. p. 824.

7) Dirac, P. A. M., The principles of Quantum Mechanics, 3d ed., Clarendon Press, Oxford (1947) $\S 52, \mathrm{p} .201-204$.

8) See Lee, ref. 1, and Wick, G. C., Rev. mod. Phys. 27 (1955) 339, esp. Section 2.

9) Weinberg, S., Phys. Rev. 102 (1956) 285. 